\title{
Hybrid Adaptive Image Restoration Method with Pixel Block Estimation and Histogram Equalization
}

\author{
Sukhwinder Singh \\ Research Scholar (ECE) \\ SBSSTC, Ferozepur-152001, \\ Punjab, India
}

\author{
Amit Grover \\ Assistant Professor (ECE) \\ SBSSTC, Ferpozepur-152001, \\ Punjab, India
}

\begin{abstract}
The process of recovering image from corrupted state is called restoration. In this paper, the combination of the neighbor based reference model and non-reference image matrix enhancement is proposed for the enhancement of the results. In this paper the restoration with image missing pixel recovery and recreation is done and non-reference restoration enhancement method is used to recover the pixel expansion problem. Then image is more enhanced by using Histogram. The experimental results have been executed over the grayscale standard images of the Lena and Barbara. The results have shown that the proposed model outperforms the existing models when evaluated on the basis of peak signal to noise ratio and mean squared error.
\end{abstract}

\section{Keywords}

Image enhancement, contrast enhancement, noise elimination, contrast adjustment.

\section{INTRODUCTION}

Image can be made more readable and clear by using Image enhancement which increase the picture quality [1]. It is essential task so that picture can be clearer and visible due to this reason Image enhancement is used in many fields involving the digital video, World Wide Web, scientific imaging and DVDs. The main task of Image enhancement is to create newest pixels from their surrounding real pixels. It generally required a change in picture measurements given by non-inter factor enhancement [2]. There are several reasons to be executed to resize an image pixels during zoom so that image pixel cannot be broken and image can easily visible [3]. With the size of user based digital photographic, users can expect to take much control over digital images. As HD television technology come the market place, so researchers are taking much interest to make best algorithm which enhance the lower picture quality to HD quality [4]. Like, Astronomical images take from probes and rovers is of lower quality so require extreme lowest transmission rate, so it is enhanced so that it can be transmitted with high resolution of data infeasible [5][6]. In the field of medical imaging, the neurologists have an ability to zoom their particular parts of brain tomography images without any breakage of pixel. A similar application has been occurred when you want to increase their resolution of image while improving it using digital imaging software. Image enhancement is also used in Web pages where low resolution images is inserted and can be enhanced when user click on it [7][8]. However, for this high quality picture has to be stored in background and can be download when it is clicked but this still consume the storage space to save storage space and communication bandwidth it would be desirable if the low resolution image is downloaded and then enhanced on the user's machine [9].

\section{LITERATURE SURVEY}

Constantina Raluca Mihalache And Mitic A Craus [10], presents the Bayesian model to (BID) blind image deconvolution problem. The novelty of model is use of sparse kernel-based model to point the spread function which lets estimated of both support and PSF shape. In proposed approach, a strong model of the blind image deconvolution errors and image prior which preserves the edges of reconstructed picture can also be used. Robustness, Sparseness, and preservation of these edges have been achieved to use priors based on Student's-t probability density function (PDF). Xue Li, Gao Shesheng, Wang Jianchao [11] non-Gaussian and nonlinear systems, the particle filtering can be much effective which is difficult to choose the vital diverges and distribution function more greatly. With this problem, paper represents the robust regularized unscented particle filtering to increase the performance of filtering. The algorithmic program is suitable to filter calculation in the nonlinear system, because controls limitations of general filter and can be used to same weight but takes merit of larger efficiency of the unscented the regularized particle filtering and particle filtering. Yang-Chih Lai, Chih-Li Huo, YuHsiang Yu, Tsung-Ying Sun [12] this study concentrates on Gaussian blur estimation for blind image deconvolution (BID) issue. In BID issue, it as it were utilizes obscured picture and less data of point spread function (PSF) to re-establish the got blurred picture.

\section{PROPOSED METHODLOGY}

In this proposed model, firstly takes image from computer or other place and load the image in working memory of the image restoration model. After loaded the image, the second step will be to perform the low-rank matrix restoration (LRMR) to restore the image. The go decomposition technique used for produced the cube of original image and its decomposed matrix. After this process, the image of pixel level restoration using the non-reference restoration (NRRA). The method will recover the missing pixel regions and will recreate the missing pixels. Then the image super pixel enhancement (Histogram Equalization) method also used to recover the pixel enlargement problem occurred through image de-noising and image restoration processes. Then, no. of performance parameters would be obtained in the form of mean squared error (MSE), peak signal to noise ratio (PSNR) and Structural similarity (SSIM). The performance of the proposed model will be completely studied in order to conclude the findings and the comparison of the proposed and existing models will be performed to increase the improvement in the performance of the proposed model. Then image is more enhanced by using Histogram. 


\subsection{Low-Rank matrix recovery model}

The LRMR technique compares the image matrix with the approximation matrix of the image to get out the irregularity within the image knowledge. The irregularity matrix is termed the error matrix and subtracted from the first image to revive the image. So remove these matrix noise or error, than we used the HSI restoration technique. For restoration process, the low-rank matrix recovery (LRMR) technique is used which can simultaneously remove Gaussian noise, impulse noise, dead pixels or lines, and stripes.

\subsection{Multi-dimensional Non reference restoration pixel enhancement Algorithm}

The image would undergo the pixel level restoration using the Non reference restoration (NRRA). The method will recover the missing pixel regions and will recreate the missing pixels. Then the image super pixel enhancement method will be used to recover the pixel expansion problem happen through image de-noising and restoration processes. In this case, it's necessary to recover the remaining pixels employing a nonreference recovery technique.

\subsection{Histogram enhancement Algorithm}

The proposed model is the amalgamation of the major image quality enhancement or restoration methods of non-reference pixel enhancement and matrix regularization method and lowrank matrix recovery. All of the latter methods has been combined for the purpose of improvement in the image quality enhancement up to the desired level and improved than the previous methods. Further the image is more enhanced by using Histogram method. The Histogram method adjust the image contrast. Histogram increase the global contrast of image, mainly when the data of the image is represented by close contrast value. By this adjustment, the intensities can be better distributed on the histogram. So histogram method produced best image quality as compare to Low Rank matrix recovery model and Non reference restoration pixel enhancement Algorithm

\section{RESULTS ANALYSIS}

The first challenge was to evaluate the performance of enhancement methods available for our research. For increase the performance of enhancement methods over the different images. The performance evaluation survey has helped to mark the difference between all of the shortlisted enhancement methods in order to obtain the best out of the given list. The results have been obtained in the form of resulting images and the performance parameters of image quality in the form of similarity and dissimilarity

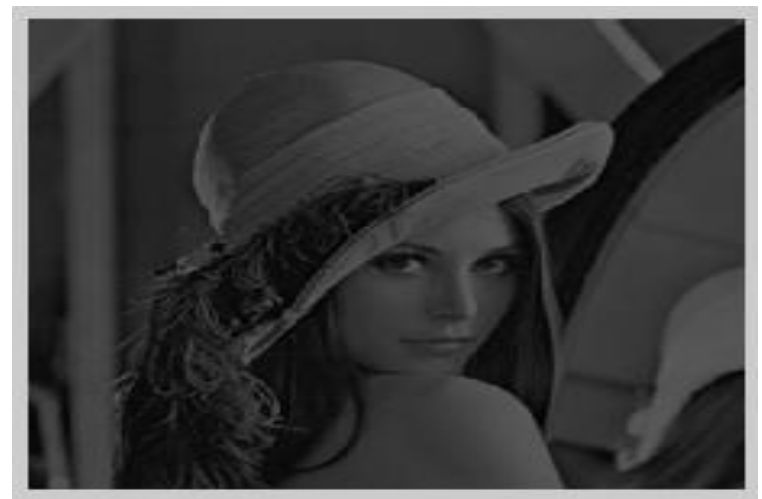

Figure1: Original Lena picture

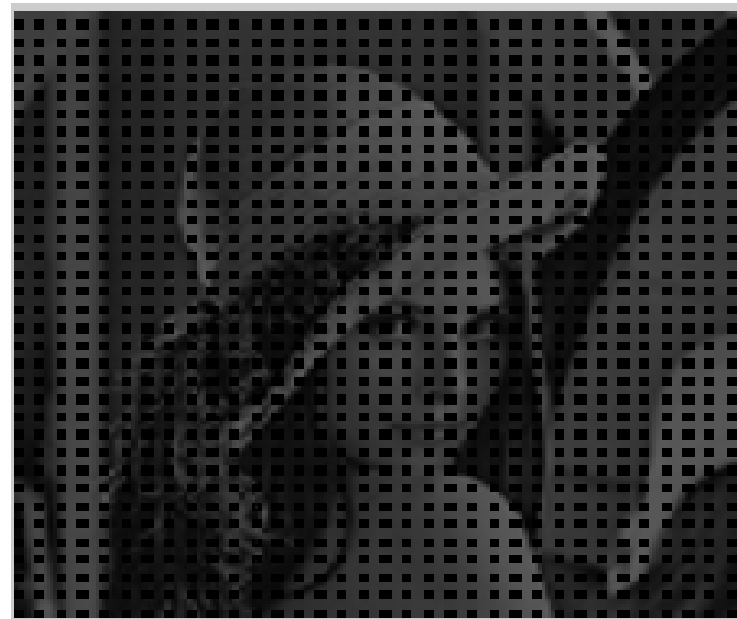

Figure 2: Image Enhancement results using LRMR.

The image (Figure 2) shows the results obtained after the wavelet based enhancement method.

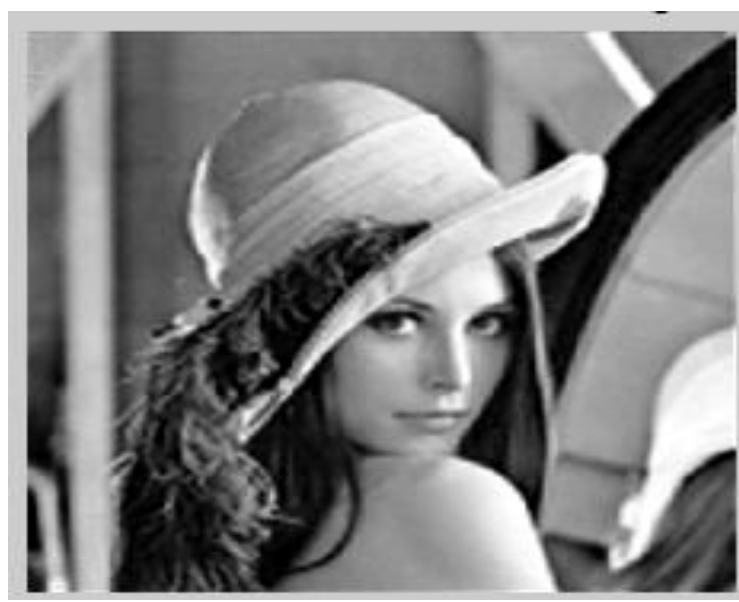

Figure 3: Image enhancement result obtained using Combined LRMR\&NRRA

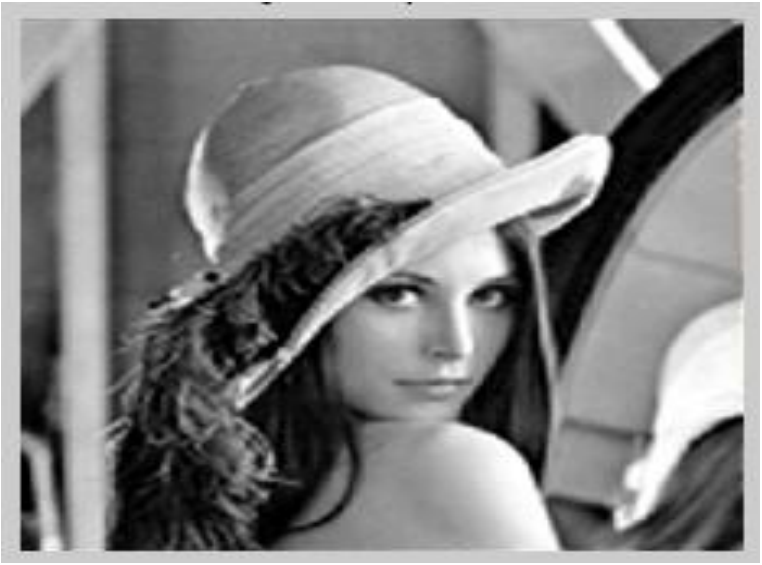

Figure 4: Image enhancement using Histogram 


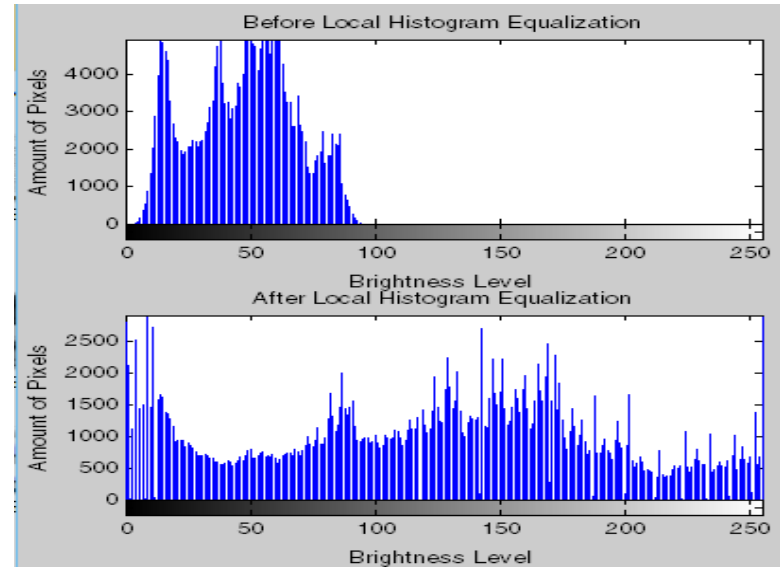

Figure 5: Histogram Pixel and Brightness Level

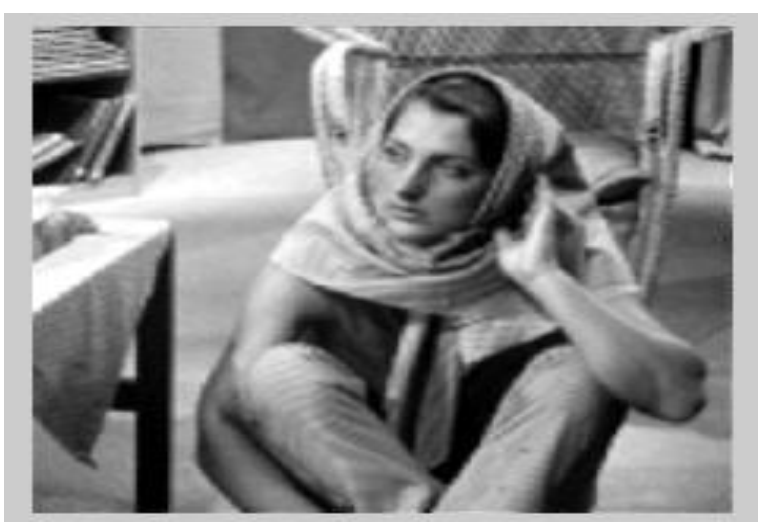

Figure 6: Original Barbara picture

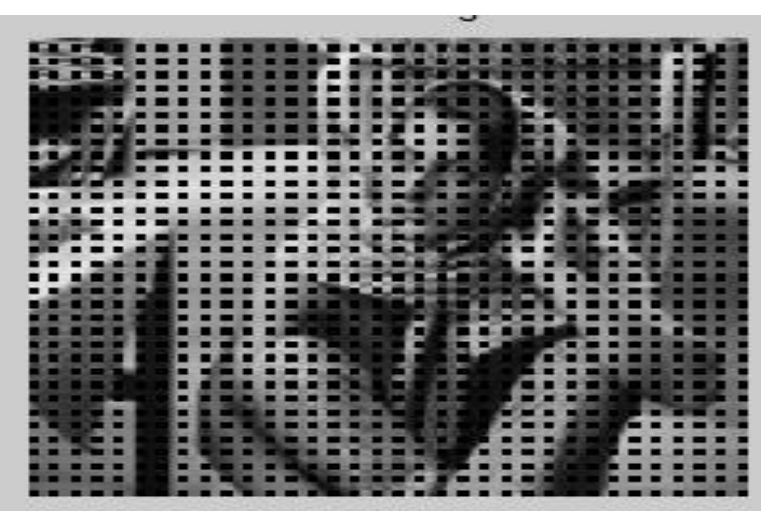

Figure 7: Image Enhancement results using LRMR

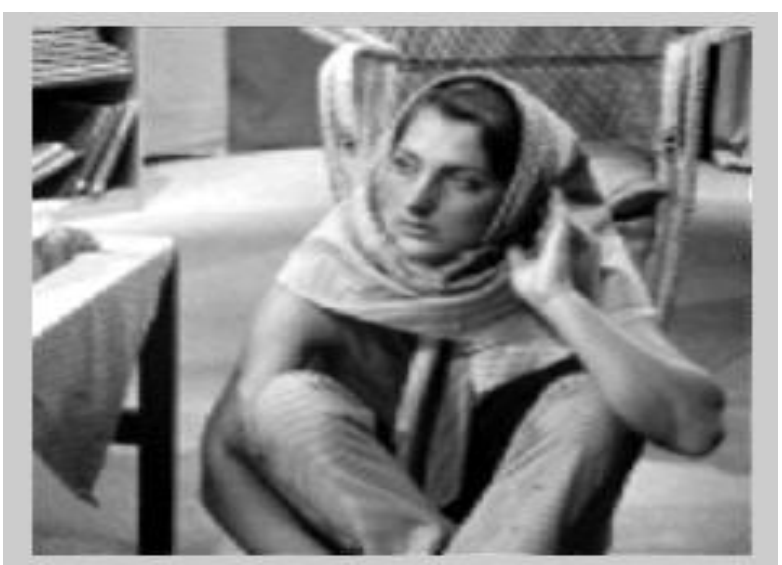

Figure 8: combined LRMR\&NRRA image

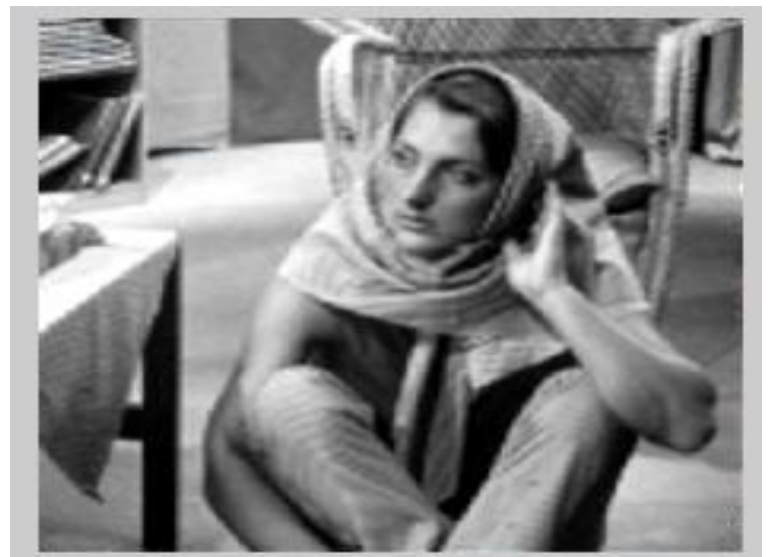

Figure9: Image Enhancement Using Histogram Equalize

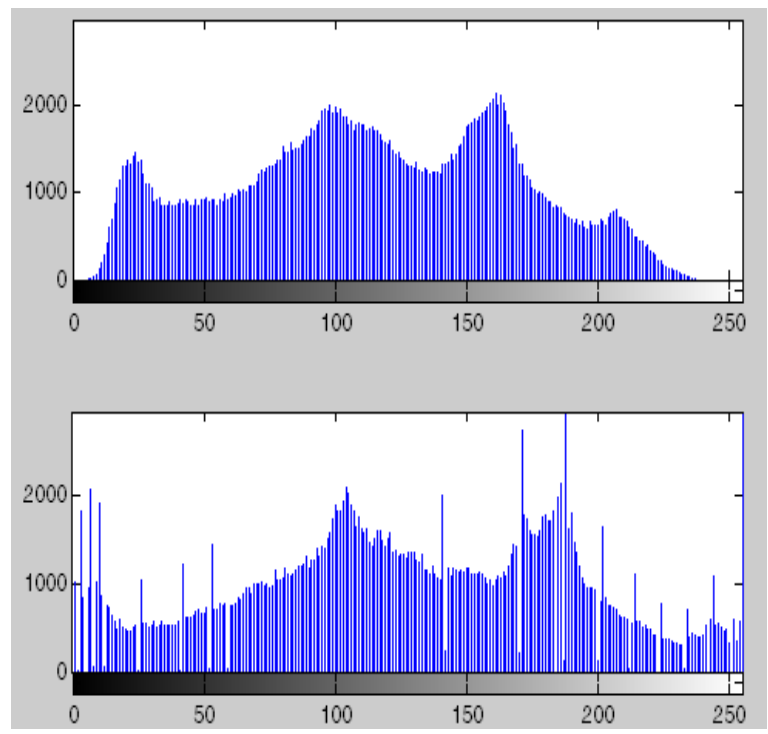

Figure10: Histogram Pixel and Brightness Level

In this proposed model the low-rank matrix restoration (LRMR) method which restore the image. This method used to create the cube of original image and its decomposed matrices. LRMR method produced matrix form images. Afterwards, the image would undergo the pixel level restoration using the Non reference restoration (NRRA). The method will recover the missing pixel regions and will recreate the missing pixels. Then Histogram Equalization method used to recover the pixel enlargement problem induced by image de-noising and restoration processes. The Histogram method increase the image contrast and increase the image quality as compare to other method. Then, various performance parameters would be obtained in the form of mean squared error (MSE), peak signal to noise ratio (PSNR) and Structural similarity (SSIM). In this research work PSNR and MSE are used to measure the quality of an image. In this paper the structural similarity used for measure the similarity between two images. The SIMM structure is improve on traditional method like peak signal to noise ratio and mean square error.

\section{RESULTS}

The results obtained from the standard images have been evaluated in table no. 1 and table no. 2. The results have been obtained in the form of PSNR, MSE and SIMM. The table no.1 elaborates the results over all of the standard images. The table no. 1 has been collected over the standard images in the grayscale color domain 
Table 1. The evolution of standard image dataset from proposed model

\begin{tabular}{|c|c|c|c|}
\hline OBJECT & \multicolumn{3}{|c|}{ LEENA } \\
\hline METHOD & LRMR & NRRA & HISTOGRAM \\
\hline PSNR & 21.203 & 42.299 & 68.992 \\
& & & 0.998 \\
\hline MSE & 0.999 & 0.998 & 0.4252 \\
\hline SIMM & 0.7901 & 0.4251 & \\
\hline
\end{tabular}

Table 2. The evolution of standard image dataset from proposed model

\begin{tabular}{|c|c|c|c|}
\hline OBJECT & \multicolumn{3}{|c|}{ BARBARA } \\
\hline METHOD & LRMR & NRRA & HISTOGRAM \\
\hline PSNR & 13.507 & 26.865 & 53.723 \\
\hline MSE & 1.000 & 1.000 & 0.998 \\
\hline SIMM & 0.8928 & 0.3717 & 0.3779 \\
\hline
\end{tabular}

Table $1 \& 2$ defines the performance measures in the form of PSNR MSE and SIMM. After the application of the proposed method over the standard images of Lena and Barbara.

\section{CONCLUSION}

The proposed model is based upon the image quality improvement using the hybrid image quality enhancement method. All the later methods have been combined for the purpose of improvement in the image quality enhancement up to the desired level. Further the image is more enhanced by using Histogram method. The proposed model has been tested with several prominent images, which includes the Lena and Barbara images. The results have been obtained in the form of peak signal to noise ratio and mean squared error. In this analysis,PSNR and MSE are used to measure the standard of a picture. Peak signal to noise ratio (PSNR) and Mean square error (MSE) are enforced so as to get some quantitative results for comparison of wavelet based improvement techniques. SIMM process is used for measuring the similarity between two images and improve on traditional method like PSNR and MSE.

\section{REFERENCES}

[1] A.Gotchev, J. Vesma, T. Saramaki and K. Egiazarian," Digital image re sampling by modified B-spline functions,"IEEE Nordic Signal Processing symposium, pp. 259-262, June 2000.

[2] A. M. Darwish, M. S. Bedair and S.I. Shaheen, "Adaptive resampling algorithm for image enhancement", IEEE Proc. Vis. Image Signal Process, vol. 144, pp 207-212 No. 4, August 1997.

[3] Andera Giachetti and Nicola Asuni, "Real-Time artifact free Image up scaling", IEEE Transactions on Image Processing, vol. 20 No. 10, October 2011.

[4] B. S. Morse and D. Schwartzwald,"Image magnification using level-set reconstruction", Proc. IEEE Int. Cof. Computer Vision Pattern Recognition, vol.3, pp. 333340, 2001

[5] Buades, Antoni, Bartomeu Coll, and Jean-Michel Morel. "Nonlocal image and movie denoising." International journal of computer vision 76.2, pp. 123-139, 2008

[6] C. B. Atkins, C. A. Bouman and J. P. Allebach, "Optimal image scaling using pixel classification", Proc. IEEE Int Conf. Image Processing, vol. 3, pp. 864-867, 2001.

[7] Concus, P., Golub, G. H., \& O'Leary,' D. P. A generalized conjugate gradient method for the numerical Solution of elliptic partial differential equations: Computer Science Department, School of Humanities and Sciences. : Stanford University, 1976

[8] Gonzalez, Rafael C. "RE woods, Digital Image Processing." Addison-Wesely Publishing Company (1992).

[9] H. chen and G. E. Ford,"An FIR enhancement filter Design method based on properties of Human Vision", Proc. IEEE Int. Conf. Image Processing, vol.3, PP.581585, November 1994.

[10] Mihalache, Constantina Raluca, and Mitica Craus. "Neural network and fuzzy membership functions based edge detection for digital images." System Theory, Control and Computing (ICSTCC), 16th International Conference on. IEEE, PP 1-6, 2012.

[11] Xue Li, GaoShesheng, Wang Jianchao, "Research on Robust Unscented Regularized Particle Filtering," IEEE, pp. 790-793, 2010.

[12] Lai, Yang-Chih, et al. "PSO-based estimation for Gaussian blur in blind image deconvolution problem." Fuzzy Systems (FUZZ), 2011 IEEE International Conference on. IEEE, PP 1143-1148, 2011 